\title{
Energy-Aware Utility Regions: Multiple Access Pareto Boundary
}

\author{
Eduard Jorswieck, Holger Boche, and Siddharth Naik
}

\begin{abstract}
Power management and energy-aware communications systems have become increasingly important in mobile computing as well as mobile communications. In future wireless communication systems, the energy efficiency of terminals and base stations has to be improved significantly. Therefore, we propose a new utility function, which is the difference of the capacity and a weighted power cost term. The generally used individual power constraint is removed. Next, the utility region for single-antenna and multi-antenna multiple access channels is characterized. We show using basic principles that the singleinput single-output (SISO) multiple-access channel (MAC) utility region is convex and provide a closed form expression for its Pareto boundary. We need the Pareto boundary to compute efficient operating points. Furthermore, the extension to multiple antenna channels is indicated by an iterative algorithm for weighted sum utility maximization in multiple-input single-output (MISO) and multiple-input multiple-output (MIMO) MAC. All discussed results are illustrated by numerical simulations.
\end{abstract}

Index Terms-Multiple access channel, utility region, convex optimization, multiple-antenna.

\section{INTRODUCTION}

$\mathbf{P}$ OWER management and energy efficient communication is an important topic in future mobile communications and computing systems. Currently $0.14 \%$ of the carbon emissions are contributed by the mobile telecommunications industry[1]. In order to improve the situation, three different approaches can be adopted: 1 . development of better energy sources, 2. network and site optimization, and 3. improved algorithms at physical and multiple access layer. The first approach lies outside the scope of the current paper and in a different community. The second approach is undertaken in [2] where a energy efficient network protocol is proposed. In [3] an extension and simplification of the protocol is proposed. Still at the network layer, a framework for computation of the capacity of ad-hoc networks under energy constraints is proposed in [4]. Finally, in [5], a Mobile Backbone Network Power Saving protocol is developed to reduce energy consumption. A lot of further recent work on wireless sensor networks considers energy-aware routing and flow control (e.g. [6]-[8]).

Manuscript received March 26, 2009; revised February 3, 2010; accepted February 26, 2010. The associate editor coordinating the review of this paper and approving it for publication was E. Hossain.

E. Jorswieck is with the Dresden University of Technology, Communications Laboratory, Chair of Communication Theory, Dresden, Germany (email: eduard.jorswieck@tu-dresden.de).

H. Boche and S. Naik are with the Fraunhofer Institute for Telecommunications, Berlin, Germany (e-mail: \{boche, naik\}@hhi.de).

This work is supported in part by the German Federal State of Saxony in the Excellence Cluster Cool Silicon in the framework of the project Cool Cellular under grant number 14056/2367.

Digital Object Identifier 10.1109/TWC.2010.07.090437
One example for the third approach is [9] where the energy efficiency of point-to-point communication systems is improved by sophisticated adaptation strategies. A coding theoretic approach is proposed in [10] where 'green codes' for energy efficient short-range communications are developed. The multiuser scenario is more difficult because of the conflicting interests of competing users and the resulting multi-criteria objective function. However, fewer results are available for energy-aware resource allocation at the physical and multiple access layer. Therefore, there are recent proposals to define a utility function which incorporates the cost of transmission, e.g., the price of spending power is considered in a binary variable in [11] and as an inverse factor in [12].

The utility region consists of utility vectors that are achievable by a certain set of resource allocation and transmit strategy parameters. In order to operate the system in an efficient way, the Pareto boundary of the utility region needs to be well understood. In Figure 1, we show a typical utility region - the rate region of the SISO MAC with successive interference cancellation (SIC) at the base station [13] under individual power constraints. The typical polymatroidal structure can be observed. The line between the operating points in which both users apply maximum transmit power and different decoding orders are applied, is achieved by rate splitting [14]. All points on this line are Pareto optimal. The points on the vertical and horizontal lines are achieved by a fixed decoding order and the user who is decoded last varies its transmit power between zero and maximum power. These points are called weakly Pareto optimal.

On the Pareto boundary, different efficient operating points can be adjusted depending on the fairness criteria, e.g., the maximum sum utility (from an operator's point of view), proportional fair utility (from a long-term fairness perspective), or the max-min fair utility point (from a short-term user perspective).

In the current paper, we propose an energy-centric point of view and define a utility function which is the difference of the individual capacity and an individually weighted power cost term. The weights contain context information of the specific user and could include battery or quality-of-service (QoS) information. Note that in [15] a similar utility function is proposed to improve the efficiency of the non-cooperative Nash equilibrium. The difference of the current work is that we characterize the Pareto boundary and do a centralized (cooperative) optimization of transmit strategies and power allocation. Using very basic principles, we provide a complete characterization of the SISO MAC utility region.

The contribution and the organization of the paper are as follows:

1) The system model, the proposed energy-aware utility 


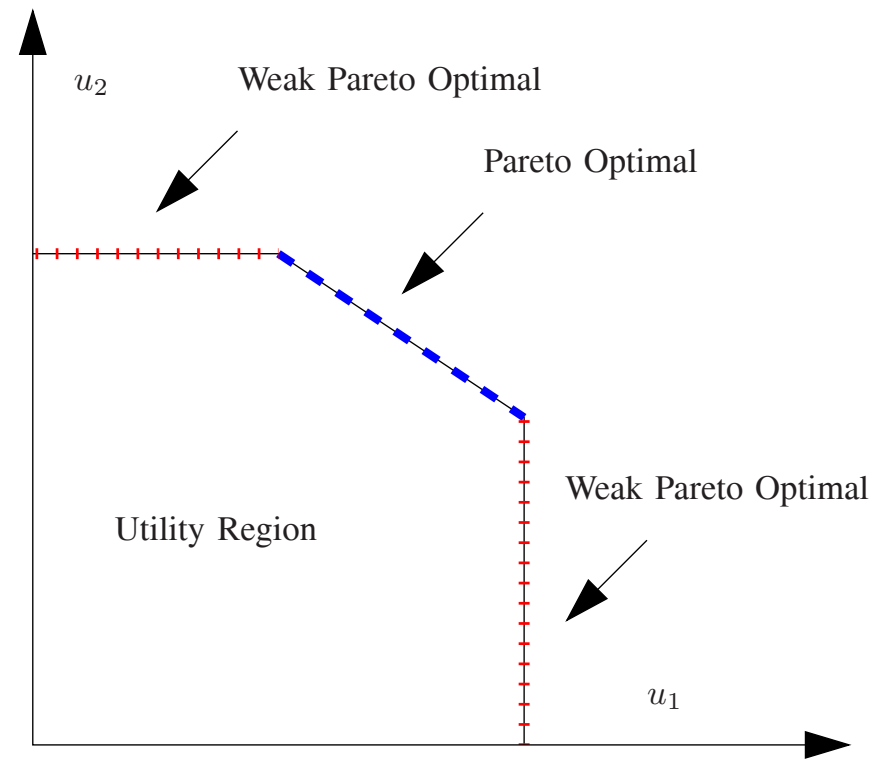

Fig. 1. Example utility region - SISO MAC with SIC.

function, and the term Pareto-optimality are explained in Section II.

2) The main results for the SISO MAC are collected in Section III. First, the Pareto optimal power allocation is characterized. Next an efficient algorithm that maximizes the weighted sum utility is derived. Finally, we show that the utility region is convex.

3) The main results and differences for the SIMO MAC are derived in Section IV. It is shown for the two user case that the region is not convex. An algorithm for sum utility maximization is derived and low- and high-signalto-noise-ratio (SNR) behavior is characterized.

4) The MIMO MAC case is studied in Section V. We propose a simple, however an efficient iterative algorithm to solve the weighed sum utility maximization problem.

The paper is concluded in Section VI.

\section{A. Notation}

Vectors are denoted in bold letters $\boldsymbol{x}$. Matrices are written in bold capital letters $\boldsymbol{H}$. Transpose is $[\cdot]^{T}$, and the conjugate transpose is $[\cdot]^{H}$. The matrix (pseudo) inverse is denoted by $[\cdot]^{-1} \cdot \operatorname{tr}(\boldsymbol{A})$ denotes the trace of the matrix $\boldsymbol{A}$, i.e. $\operatorname{tr}(\boldsymbol{A})=$ $\sum_{k=1}^{n} \boldsymbol{A}_{k, k} . \omega_{i}(\boldsymbol{A})$ is the $i$ th eigenvalue of the matrix $\boldsymbol{A}$. $\omega_{\max }(\boldsymbol{A})$ is the largest eigenvalue of the matrix $\boldsymbol{A} \cdot \mathcal{R}(x)$ denotes the real part of the complex variable $x$. $\mathbb{C}_{+}^{n}$ denotes the set of positive semidefinite matrices. $\|\boldsymbol{a}\|$ is the $l_{1}$-norm, i.e. $\|\boldsymbol{a}\|=\sum_{i=1}^{n}\left|a_{i}\right|$. The order for matrices is denoted by $\boldsymbol{A} \succeq \boldsymbol{B}$ and this means that the difference $\boldsymbol{A}-\boldsymbol{B}$ is positive semi-definite. $\operatorname{diag}(\boldsymbol{A})$ is the vector with diagonal entries of $\boldsymbol{A}$ and $\operatorname{Diag}(\boldsymbol{a})$ is a matrix with entries of the vector $\boldsymbol{a}$ on the diagonal.

\section{PReliminaries}

\section{A. System Model and Channel Model}

We consider the MAC, i.e., the uplink, where a number of $K$ terminals wants to transmit their information to a base station. In the following, we distinguish between three cases depending on the number of antennas at the terminals or the base. In the single-input single-output (SISO) MAC all terminals as well as the base have a single transmit or receive antenna, respecively. The received signal at the base station is given by

$$
y=\sum_{k=1}^{K} x_{k} a_{k}+n
$$

where $x_{k}$ is the transmitted signal of user $k$ and $a_{k}$ corresponds to the complex channel coefficient of user $k$. We denote the channel gain of user $k$ by $\alpha_{k}=\left|a_{k}\right|^{2}$. The additive white Gaussian noise $n$ is zero-mean complex Gaussian distributed with variance $\sigma_{n}^{2}$. If the base station has multiple receive antennas, we talk about the single-input multiple-output (SIMO) $\mathrm{MAC}$, the received signal vector is given by

$$
\boldsymbol{y}=\sum_{k=1}^{K} x_{k} \boldsymbol{h}_{k}+\boldsymbol{n}
$$

where $x_{k}$ is the transmitted signal of user $k$ and $\boldsymbol{h}_{k}$ is the vector channel from user $k$ to the receive antennas at the base station. The additive white Gaussian noise vector at the base is zero-mean complex Gaussian distributed with covariance matrix $\sigma_{n}^{2} \boldsymbol{I}$. In both cases (1) and (2), we denote the transmit power of user $k$ as $p_{k}$, i.e., $\mathbb{E}\left[\left|x_{k}\right|^{2}\right]=p_{k}$. Finally, the case in which the users as well as the base have multiple antennas is called multiple-input multiple-output (MIMO) MAC. The received vector at the base station is given by

$$
\boldsymbol{y}=\sum_{k=1}^{K} \boldsymbol{H}_{k} \boldsymbol{x}_{k}+\boldsymbol{n}
$$

where $\boldsymbol{x}_{k}$ is the transmitted signal vector of user $k$ with transmit covariance matrix $\boldsymbol{Q}_{k}=\mathbb{E}\left[\boldsymbol{x}_{k} \boldsymbol{x}_{k}^{H}\right]$ and the channel matrix $\boldsymbol{H}_{k}$ collects the channel realizations from all transmit antennas of user $k$ to the base. The additive white Gaussian noise vector at the base is zero-mean complex Gaussian distributed with covariance matrix $\sigma_{n}^{2} \boldsymbol{I}$. The transmit power of user $k$ is $p_{k}=\operatorname{tr}\left(\boldsymbol{Q}_{k}\right)$.

In all scenarios, we assume that the base station has perfect channel state information (CSI). Further, we assume that the transmitters are informed about their transmit strategies (either power allocation or transmit covariance matrix) by the base station via a control channel.

\section{B. Energy-Aware Utilty Function}

Define the utility of user $k$ for some $\mu_{k} \geq 0$ as

$$
u_{k}(\boldsymbol{p})=r_{k}(\boldsymbol{p})-\mu_{k} p_{k}
$$

where $r_{k}(\boldsymbol{p})$ is the achievable transmission rate of user $k$ in one of our system environments described above and $\boldsymbol{p}$ is the power allocation vector $\boldsymbol{p}=\left[p_{1}, \ldots, p_{K}\right]$.

Let us illustrate the operational meaning of the utility function in (4) in the single-antenna scenario. We denote the single-user rate without interference as $r(p)=\log \left(1+\rho \alpha_{k} p\right)$ where $\rho$ is the inverse noise power and $\alpha_{k}$ is the channel power. The corresponding utility is $u(p)=\log \left(1+\rho \alpha_{k} p\right)-\mu p$. In contrast to the pure rate function, the utility in (4) takes the cost of transmission into account. If some power $p_{k}$ is 


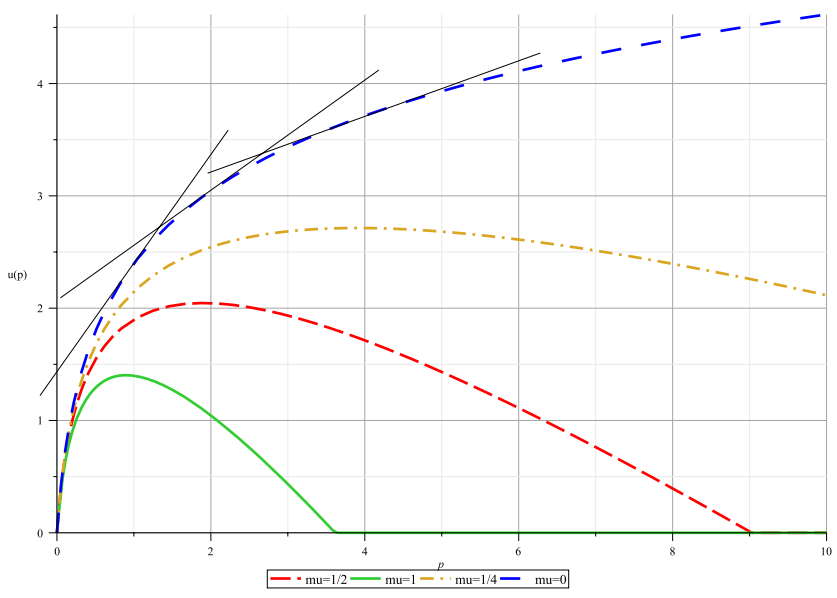

Fig. 2. Utility function $u(P)=\log (1+10 p)-\mu p$ over $p$ in linear scale.

spent for transmission it linearly reduces the utility by a factor $\mu_{k} p_{k}$. Let us call $\mu_{k}$ the power price of user $k$. This interpretation is illustrated by the following scenario: if $\mu_{k}$ gets too large compared to the $\log \left(1+\rho \alpha_{k} p_{k}\right)$ term, i.e., the costs of allocating power are larger than the gain by reliable transmission, the user will switch off, i.e., $p_{k}=0$, until the gain by transmission is larger than the cost. The key design quantities are the rate $r_{k}[\mathrm{bit} / \mathrm{s}]$, the transmit power $p_{k}$ [W], and the price per unit of power $\mu_{k}[\mathrm{bit} / \mathrm{s} / \mathrm{W}]$.

The variable $\mu_{k}$ occurs also in the optimization of the rates with sum power constraints as Lagrangian dual variables [16]. In this context, $\mu_{k}$ does not posses the meaning explained above. However, the resulting iterative algorithm could have a similar form. The difference to [16] is that we either completely characterize the Pareto boundary (SISO MAC) or propose an algorithm for the weighed utility maximization (MIMO MAC) which is a non-trivial generalization of [16].

A similar utility function is proposed in [17] for singleantenna systems and used to characterize the Nash equilibrium for the non-cooperative power control game. Later in [18] the approach is extended to multiple antenna channels in a related non-cooperative game-theoretic setting.

In Figure 2, the utility function is shown for different values of $\mu_{k}$. By choosing the power price $\mu_{k}$ carefully, we can force the user to operate in the energy efficient power regime. The rate function scales logarithmically with the power. From an energy efficiency point of view, the power regime in which the rate scales linearly with the power is of interest.

Note, that the utility function $u$ is unimodal, i.e. monotonic increasing up to the maximum and then monotonic decreasing, and concave with respect to $p$. The optimal single-user operating point is easily found by computing the first derivative of the utility with respect to $p_{k}$ and solving to obtain

$$
p_{k}^{*}=\left(\frac{1}{\mu_{k}}-\frac{1}{\rho \alpha_{k}}\right)^{+} \text {. }
$$

The corresponding achievable utility is

$$
u_{k}\left(p_{k}^{*}\right)=\log \left(\frac{\rho \alpha_{k}}{\mu_{k}}\right)^{+}-\left[1-\frac{\mu_{k}}{\rho \alpha_{k}}\right]^{+} .
$$

Note that $u_{k}\left(p_{k}^{*}\right)$ in (6) decreases with $\mu_{k}$ and increases with $\rho \alpha_{k}$. Furthermore, the optimal operating point in (5)

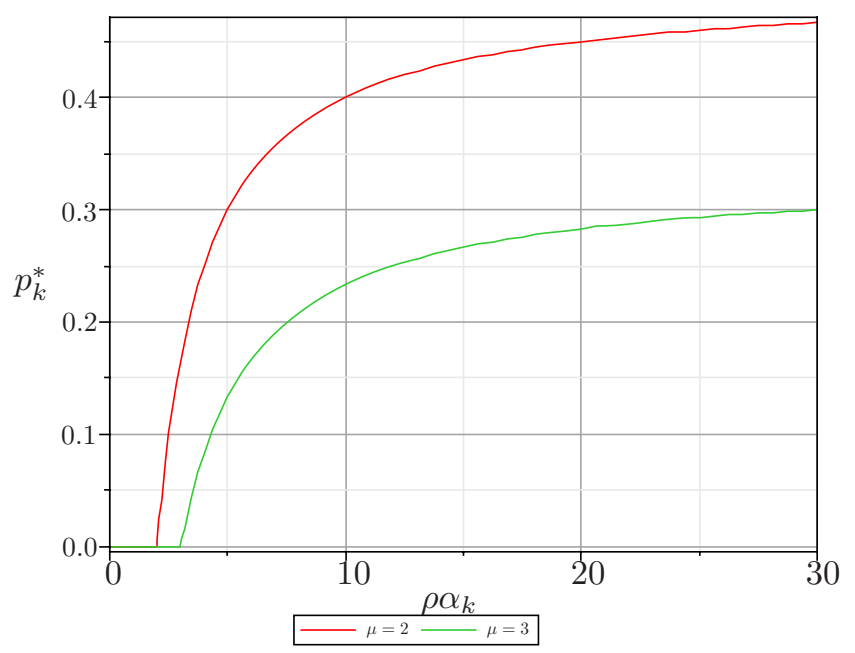

Fig. 3. Optimal single-user power allocation $p_{k}$ as a function of $\rho \alpha_{k}$.

TABLE I

COMPARISON: CAPACITY REGION AND UTILITY REGION WITH PRICING

\begin{tabular}{l|l|l} 
& Capacity without pricing & Utility including pricing \\
\hline $\begin{array}{l}\text { Power con- } \\
\text { straints }\end{array}$ & $\begin{array}{l}\text { Explicit transmit power } \\
\text { constraints }\end{array}$ & $\begin{array}{l}\text { Implicit power constraint } \\
\text { by prices }\end{array}$ \\
\hline SISO region & $\begin{array}{l}\text { Decoding order depends } \\
\text { only on weights, }\end{array}$ & $\begin{array}{l}\text { Decoding order depends on } \\
\text { the effective channels in- } \\
\text { cluding pricing }\end{array}$ \\
\hline $\begin{array}{l}\text { MIMO } \\
\text { region and } \\
\text { optimiza- } \\
\text { tion }\end{array}$ & $\begin{array}{l}\text { Iterative waterfilling (IWF) } \\
\text { for sum capacity maxi- } \\
\text { mization }\end{array}$ & $\begin{array}{l}\text { Inner optimization with } \\
\text { IWF, outer optimization } \\
\text { for sum transmit power }\end{array}$ \\
$\begin{array}{l}\text { Single-user range depends } \\
\text { on power constraint and } \\
\text { channels }\end{array}$ & $\begin{array}{l}\text { Single-user range depends } \\
\text { on channels and prices }\end{array}$
\end{tabular}

corresponds to an effective power constraint of $\frac{1}{\mu_{k}}$ illustrated in Figure 3 where the power as a function of $\rho \alpha_{k}$ is shown. From (6) follows that zero power is allocated if the slope of the rate $r_{k}$ at zero is smaller than $\mu_{k}$, i.e., if the power price is too large compared to the rate gain.

From (5) and Figure 3 follows that for all $\mu_{k}>0$ an implicit power constraint of $\frac{1}{\mu}$ is given. Therefore, we will not impose an additional explicit power constraint in the following derivation.

Remark 1: It is a remarkable fact, that we do not need an explicit individual or sum power constraint in the singleuser scenario. Instead by using the linear power price the system automatically operates in an efficient regime. As a convenient byproduct the SNR cannot be defined explicitly and the dependency of the result on the SNR is expressed indirectly by $\rho$ which is the inverse noise variance. We note that in interference networks, the relation between the price $\mu_{k}$ and the maximum transmit power of user $k$ is more complicated and explicit power constraints could be applied.

The difference of the utility function in (4) compared to the rate function $r_{k}$ with additional transmit power constraint is that the utility function forces the user to operate in the power efficient regime. In Table I, the relationship between the capacity region without pricing and the proposed utility function and its corresponding region are summarized. 


\section{Pareto Optimality}

Next, we study the efficient operating points in the multiuser utility region. The achievable utility region is defined as

$$
\mathcal{U} \triangleq \bigcup_{\boldsymbol{p} \geq 0}\left\{u_{1}(\boldsymbol{p}), \ldots, u_{K}(\boldsymbol{p})\right\} \subset \mathbb{R}_{+}^{K} .
$$

We define the Pareto optimality of an operating point, i.e., utility tuple, as follows.

Definition 1: A utility tuple $\left(u_{1}, \ldots, u_{K}\right) \in \mathcal{U}$ is Pareto optimal if there is no other tuple $\left(q_{1}, \ldots, q_{K}\right) \in \mathcal{U}$ with $\left(q_{1}, \ldots, q_{K}\right) \geq\left(u_{1}, \ldots, u_{K}\right)$ and $\left(q_{1}, \ldots, q_{K}\right) \neq\left(u_{1}, \ldots, u_{K}\right)$ (the inequality is component-wise).

As shown in Figure 1, all other boundary points of the utility region are weak Pareto optimal. At a weak Pareto optimal point it is possible to increase the utility of one user without decreasing the other users. We are not interested in these inefficient operating points because at those operating points we could improve the utility of one user without reducing the utility of another user.

\section{Utility REGION - SISO MAC}

It is well known that the capacity region of the SISO Gaussian MAC can be theoretically achieved with SIC at the base station and Gaussian transmit signals. Since our utility function in (4) is linear in the user rates, we are interested in maximizing the individual rates. Therefore, we consider the $K$ user Gaussian MAC with SIC at the base station.

Assume a decoding order of $K \rightarrow K-1 \rightarrow \ldots \rightarrow 1$. The achievable rate of user $k$ is given by

$$
r_{k}(\boldsymbol{p})=\log \left(1+\frac{\rho \alpha_{k} p_{k}}{1+\rho \sum_{l=1}^{k-1} \alpha_{l} p_{l}}\right) .
$$

Observe that the individual user rates depend on the SIC decoding order.

\section{A. Pareto Boundary of the SISO MAC}

The next result parameterizes the Pareto boundary of the utility $\mathcal{U}$ in (7). We assume a decoding order of $K \rightarrow K-1 \rightarrow$ $\ldots \rightarrow 1$ induced by

$$
\frac{\alpha_{1}}{\mu_{1}} \leq \frac{\alpha_{2}}{\mu_{2}} \leq \ldots \leq \frac{\alpha_{K}}{\mu_{K}}
$$

The reason for the optimal ordering in (9) is that we could define a new power allocation $\tilde{p}_{k}=\mu_{k} p_{k}$ and rewrite the utility function in (4) as

$$
u_{k}(\tilde{\boldsymbol{p}})=\log \left(1+\frac{\rho \frac{\alpha_{k}}{\mu_{k}} \tilde{p}_{k}}{1+\rho \sum_{l=1}^{k-1} \frac{\alpha_{l}}{\mu_{l}} \tilde{p}_{l}}\right)-\tilde{p}_{k}
$$

with the effective channels $\left(\frac{\alpha_{1}}{\mu_{1}}, \frac{\alpha_{2}}{\mu_{2}}, \ldots, \frac{\alpha_{K}}{\mu_{K}}\right)$. Therefore, in (9), the effective channels are ordered in increasing order. We define the following set of power allocations

$$
\begin{aligned}
& \mathcal{P}=\left\{\quad\left(p_{1}, p_{2}, \ldots, p_{K}\right) \in \mathbb{R}_{+}^{K}:\right. \\
& 0 \leq p_{1} \leq\left(\frac{1}{\mu_{1}}-\frac{1}{\rho \alpha_{1}}\right)^{+} \text {, } \\
& 0 \leq p_{2} \leq\left(\frac{1}{\mu_{2}}-\frac{1}{\rho \alpha_{2}}-\frac{\alpha_{1}}{\alpha_{2}} p_{1}\right)^{+} \text {, } \\
& 0 \leq p_{k} \leq\left(\frac{1}{\mu_{k}}-\frac{1}{\rho \alpha_{k}}-\sum_{l=1}^{k-1} \frac{\alpha_{l} p_{l}}{\alpha_{k}}\right)^{+} \\
& \left.p_{K}=\left(\frac{1}{\mu_{K}}-\frac{1}{\rho \alpha_{K}}-\sum_{l=1}^{K-1} \frac{\alpha_{l} p_{l}}{\alpha_{K}}\right)^{+}\right\} .
\end{aligned}
$$

Note that $p_{K}$ is a function of $p_{1}, \ldots, p_{K-1}$.

Theorem 1: A power allocation $\boldsymbol{p}^{*}$ is Pareto optimal if and only if $\boldsymbol{p}^{*} \in \mathcal{P}$ defined in (10) with SIC decoding order in (9).

Proof: The proof consists of two parts. In the first part, we show that the optimal SIC order is characterized by (9). In the second part, we show that the power allocation in (10) corresponds exactly to all Pareto optimal utility points.

The main idea for the first part of the proof is not to study the utility region for different decoding orders but the corresponding power regions, i.e., the set of feasible power allocations $\tilde{\boldsymbol{p}}$ for the effective channels $\frac{\alpha_{k}}{\mu_{k}}$. It is sufficient to consider two users $k$ and $k+1$ and define $z=\sum_{l=1}^{k-1} \frac{\alpha_{l}}{\mu_{l}} \tilde{p}_{l}$. Define the feasible power region of user $k, k+1$ for the decoding order $k \rightarrow k+1$ as $\mathcal{P}_{k, k+1}^{k \rightarrow k+1}$ and for the other decoding order $k+1 \rightarrow k$ as $\mathcal{P}_{k, k+1}^{k+1 \rightarrow k}$. They are given by

$$
\begin{gathered}
\mathcal{P}_{k, k+1}^{k \rightarrow k+1}=\left\{\tilde{p}_{k}, \tilde{p}_{k+1} \in \mathbb{R}_{+}^{2}:\right. \\
0 \leq \tilde{p}_{k} \leq\left(1-\frac{\mu_{k}}{\rho \alpha_{k}}-\frac{\mu_{k}}{\alpha_{k}} \frac{\alpha_{k+1}}{\mu_{k+1}} \tilde{p}_{k+1}-\frac{\mu_{k}}{\alpha_{k}} z\right)^{+}, \\
\left.0 \leq \tilde{p}_{k+1} \leq\left(1-\frac{\mu_{k+1}}{\rho \alpha_{k+1}}-\frac{\mu_{k+1}}{\alpha_{k+1}} z\right)^{+}\right\}
\end{gathered}
$$

and for the other (optimal) decoding order

$$
\begin{gathered}
\mathcal{P}_{k, k+1}^{k+1 \rightarrow k}=\left\{\tilde{p}_{k}, \tilde{p}_{k+1} \in \mathbb{R}_{+}^{2}:\right. \\
0 \leq \tilde{p}_{k} \leq\left(1-\frac{\mu_{k}}{\rho \alpha_{k}}-\frac{\mu_{k}}{\alpha_{k}} z\right)^{+} \\
\left.0 \leq \tilde{p}_{k+1} \leq\left(1-\frac{\mu_{k+1}}{\rho \alpha_{k+1}}-\frac{\mu_{k+1}}{\alpha_{k+1}} \frac{\alpha_{k}}{\mu_{k}} \tilde{p}_{k}-\frac{\mu_{k+1}}{\alpha_{k+1}} z\right)^{+}\right\} .
\end{gathered}
$$

In order to prove that the decoding order $k+1 \rightarrow k$ is better than $k \rightarrow k+1$, we show that

$$
\mathcal{P}_{k, k+1}^{k \rightarrow k+1} \subseteq \mathcal{P}_{k, k+1}^{k+1 \rightarrow k}
$$

using the assumption made above that

$$
\frac{\alpha_{k+1}}{\mu_{k+1}} \frac{\mu_{k}}{\alpha_{k}} \geq 1
$$

The idea is illustrated in Figure 4.

Obviously, the two regions are described by triangles since $\tilde{p}_{k}$ depends linearly on $\tilde{p}_{k+1}$ and vice versa. Hence, it suffices to show that the two corner points $\circledast$ and $\ominus$ of $\mathcal{P}_{k, k+1}^{k+1 \rightarrow k}$ are larger than those of $\mathcal{P}_{k, k+1}^{k \rightarrow k+1}$. For the right corner $\circledast$, $\tilde{p}_{k}$ achieves its maximum value for $\tilde{p}_{k+1}=0$ in $\mathcal{P}_{k, k+1}^{k \rightarrow k+1}$ but in $\mathcal{P}_{k, k+1}^{k+1 \rightarrow k} \tilde{p}_{k}$ achieves its maximum value already for 


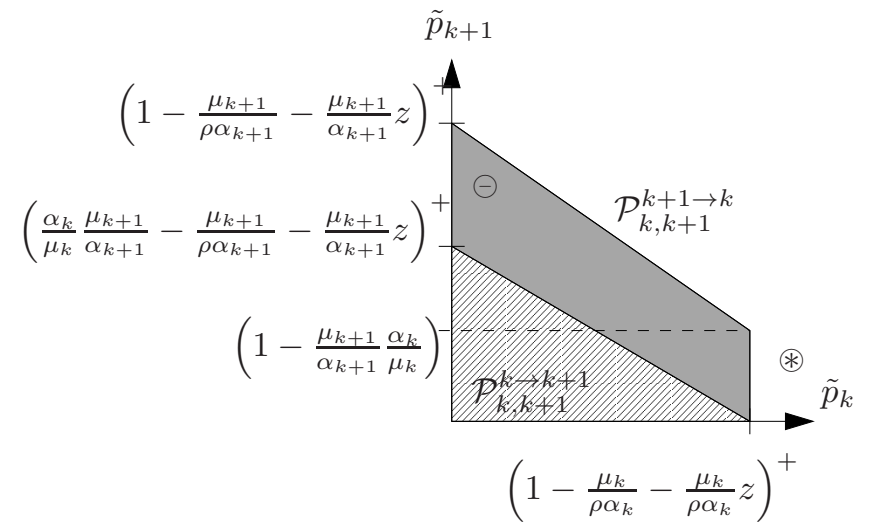

Fig. 4. Illustration of power regions $\mathcal{P}_{k, k+1}^{k \rightarrow k+1}$ and $\mathcal{P}_{k, k+1}^{k+1 \rightarrow k}$ in the proof of Theorem 1.

$\tilde{p}_{k+1}=\left(1-\frac{\mu_{k+1}}{\alpha_{k+1}} \frac{\alpha_{k}}{\mu_{k}}\right)>0$ because of (13). For the left corner $\ominus$, in $\mathcal{P}_{k, k+1}^{k \rightarrow k+1}, \tilde{p}_{k}$ is equal to zero if $\tilde{p}_{k+1} \geq$ $\left(\frac{\alpha_{k}}{\mu_{k}} \frac{\mu_{k+1}}{\alpha_{k+1}}-\frac{\mu_{k+1}}{\rho \alpha_{k+1}}-\frac{\mu_{k+1}}{\alpha_{k+1}} z\right)^{+}$, but in $\mathcal{P}_{k, k+1}^{k+1 \rightarrow k}, \tilde{p}_{k}$ is equal to zero if $\tilde{p}_{k+1} \geq\left(1-\frac{\mu_{k+1}}{\rho \alpha_{k+1}}-\frac{\mu_{k+1}}{\alpha_{k+1}} z\right)^{+}$. Therefore, decoding order $k+1 \rightarrow k$ has larger power region than decoding order $k \rightarrow k+1$. The final argument for an arbitrary permutation $\pi \neq\{K, K-1, K-2, \ldots, 2,1\}$ is that successive reordering of two neighbor indices increases the utility region.

With the Pareto optimal decoding order, the Pareto optimal power allocation can be obtained iteratively starting by the last decoded user one. With the range of $p_{k}$ the optimal power range of user $k+1$ is obtained from solving $\frac{\partial u_{k+1}(\boldsymbol{p})}{\partial p_{k+1}}=0$. In order to show that all power allocations characterized by (10) are Pareto optimal, there are two parts to prove. The first part is to show that all power allocations $\boldsymbol{p} \notin \mathcal{P}$ are not Pareto optimal. The second part is to show that all power allocations $\boldsymbol{p} \in \mathcal{P}$ are Pareto optimal.

All $\boldsymbol{p} \notin \mathcal{P}$ are not Pareto optimal: $\Longleftarrow$ We will show this direction by contradiction. We choose a power vector $\check{\boldsymbol{p}}=$ $\left[\check{p}_{1}, \ldots, \check{p}_{K}\right] \notin \mathcal{P}$. Then either one or multiple $\check{p}_{k}$ for $1 \leq k \leq$ $K-1$ lie outside the interval specified by (10) or/and $\check{p}_{K}$ is not equal to $p_{k}^{*}$.

1) Assume $\check{p}_{K} \neq\left(\frac{1}{\mu_{k}}-\frac{1}{\rho \alpha_{k}}-\sum_{l=1}^{K-1} \frac{\alpha_{l} p_{l}}{\alpha_{K}}\right)$ but all $\check{p}_{l}=$ $p_{l}^{*}$ for $1 \leq l \leq K-1$. Then we can increase the utility of user $K$, without affecting the utilities of the other users, by choosing $\check{p}_{K}=p_{K}^{*}$ because the global optimum for $u_{K}\left(p_{1}^{*}, p_{2}^{*}, \ldots, p_{K-1}^{*}, p_{k}\right)$ for fixed $p_{1}^{*}, \ldots, p_{K-1}^{*}$ is $p_{K}^{*}$ characterized in (10). Hence, $\check{\boldsymbol{p}}$ cannot be Pareto optimal.

2) At least one $\check{p}_{\ell}>p_{\ell}^{*}$ in (10) for $1 \leq \ell \leq K-1$. Then we can improve the utilities of users $\ell, \ell+1, \ldots, K$ by reducing $\check{p}_{\ell}$ to $\left(\frac{1}{\mu_{\ell}}-\frac{1}{\rho \alpha_{\ell}}-\sum_{l=1}^{\ell-1} \frac{\alpha_{l} p_{l}}{\alpha_{\ell}}\right)$. The utility of user $\ell$ is improved because the global optimum of $u_{\ell}\left(p_{1}^{*}, \ldots, p_{\ell-1}^{*}, p_{\ell}, p_{\ell+1}, \ldots, p_{K}\right)$ for fixed $p_{1}^{*}, \ldots, p_{\ell-1}^{*}$ is $p_{\ell}^{*}$. The utility of the subsequent users $\ell+1, \ldots, K$ is improved because they observe less interference from user $\ell$. Hence, $\check{\boldsymbol{p}}$ cannot be Pareto optimal.

All $\boldsymbol{p} \in \mathcal{P}$ are Pareto optimal: $\Longrightarrow$ We show this direction by contradiction, as well. Assume that there are two power vectors $\hat{\boldsymbol{p}}, \hat{\boldsymbol{q}} \in \mathcal{P}$ for which $\hat{\boldsymbol{q}}$ is not Pareto optimal, i.e., $\boldsymbol{u}(\hat{\boldsymbol{p}})>\boldsymbol{u}(\hat{\boldsymbol{q}})$, i.e., there is at least one $\ell$ with $u_{\ell}(\hat{\boldsymbol{p}})>u_{\ell}(\hat{\boldsymbol{q}})$ and $u_{k}(\hat{\boldsymbol{p}}) \geq u_{k}(\hat{\boldsymbol{q}})$ for all $1 \leq k \leq K$. Consider $u_{\ell}(\hat{\boldsymbol{p}})$ and $u_{\ell}(\hat{\boldsymbol{q}})$ : Reduce the power of user $\ell$ until $u_{\ell}(\tilde{\hat{\boldsymbol{p}}})=u_{\ell}(\hat{\boldsymbol{q}})$. Then the following inequality holds

$$
u_{\ell+k}(\tilde{\hat{\boldsymbol{p}}})>u_{\ell+k}(\hat{\boldsymbol{p}}) \geq u_{\ell+k}(\hat{\boldsymbol{q}})
$$

for all $k>0$ because user $\ell$ creates less interference for $\tilde{\hat{p}}$. Especially, we have that

$$
u_{K}(\tilde{\hat{\boldsymbol{p}}})>u_{K}(\hat{\boldsymbol{p}}) \geq u_{K}(\hat{\boldsymbol{q}})
$$

From (14) follows that the constructed $\tilde{\hat{p}} \notin \mathcal{P}$ with the property that $\boldsymbol{u}(\tilde{\hat{\boldsymbol{p}}})>\boldsymbol{u}(\hat{\boldsymbol{q}})$ for $\hat{\boldsymbol{q}} \in \mathcal{P}$ which is a contradiction to the first part of the proof. Hence, all $\boldsymbol{p} \in \mathcal{P}$ must be Pareto optimal.

Remark 2: An important special case from above is the sum utility maximization, i.e.,

$$
\max _{\boldsymbol{p} \geq 0} \log \left(1+\rho \sum_{k=1}^{K} \alpha_{k} p_{k}\right)-\sum_{k=1}^{K} \mu_{k} p_{k} .
$$

The optimization problem is a convex optimization, i.e., maximization of concave function over convex constraint set plus at least one feasible point [19]. Introduce Lagrangian multipliers $\psi_{k}$ for non-negativeness constraint, and derive the necessary and sufficient Karush-Kuhn-Tucker optimality conditions

$$
\frac{\rho \alpha_{l}}{1+\rho \sum_{k=1}^{K} \alpha_{k} p_{k}}=\mu_{l}-\psi_{l}, \quad \psi_{l} p_{l}=0, \quad \psi_{l} \geq 0 .
$$

The Lagrangian multiplier $\psi_{l}$ is zero if $p_{l}>0$, therefore it must hold for all active users $l, m \in \mathcal{A}=\left\{k: p_{k}>0\right\}$ that $\frac{\alpha_{l}}{\mu_{l}}=\frac{\alpha_{m}}{\mu_{m}}$. Therefore, the only active user is the best user corresponding with $\max _{1 \leq k \leq K} \frac{\alpha_{k}}{\mu_{k}}$. This is interesting since we do not have individual or sum power constraints.

For $K$ users, the Pareto boundary is a $K-1$ dimensional surface parameterized by the powers of $K-1$ users $\left[p_{1}, \ldots, p_{K-1}\right]$.

Corollary 1: For the decoding order in (9), the achievable utilities are characterized by

$$
\begin{aligned}
u_{1}\left(p_{1}\right)= & \log \left(1+\rho p_{1} \alpha_{1}\right)-p_{1} \mu_{1} \\
u_{2}\left(p_{1}, p_{2}\right)= & \log \left(1+\rho p_{1} \alpha_{1}+\rho p_{2} \alpha_{2}\right) \\
& -\log \left(1+\rho p_{1} \alpha_{1}\right)-\mu_{2} p_{2} \\
u_{k}\left(p_{1}, \ldots, p_{k}\right)= & \log \left(1+\rho \sum_{l=1}^{k} p_{l} \alpha_{l}\right) \\
& -\log \left(1+\rho \sum_{l=1}^{k-1} p_{l} \alpha_{l}\right)-\mu_{k} p_{k} \\
u_{K}\left(p_{1}, \ldots, p_{K-1}\right)= & \log \left(\frac{\rho \alpha_{K}}{\mu_{K}}\right)-\log \left(1+\rho \sum_{l=1}^{K-1} \alpha_{l} p_{l}\right) \\
& -\left[1-\frac{\mu_{K}}{\rho \alpha_{K}}-\sum_{l=1}^{K-1} \frac{\mu_{K} \alpha_{l}}{\alpha_{K}}\right]^{+} .(17)
\end{aligned}
$$

The utility region can also be expressed in terms of the 


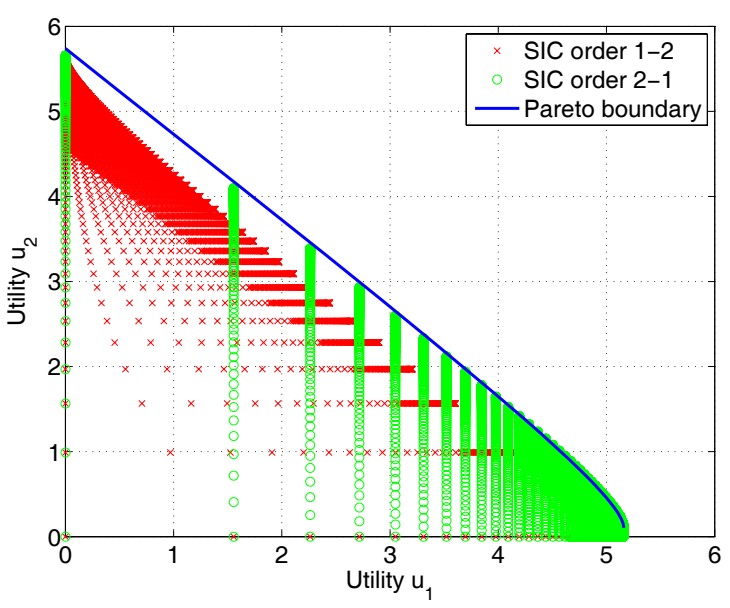

Fig. 5. Two-user MAC utility region $\mathcal{U}$ and its Pareto boundary for $\alpha_{1}=$ $2, \alpha_{2}=1, \mu_{1}=3, \mu_{2}=1, \rho=20 d B$.

following equalities

$$
\begin{array}{r}
\sum_{l=1}^{k} u_{l}(\boldsymbol{p})=\log \left(1+\rho \sum_{l=1}^{k} p_{l} \alpha_{l}\right)-\sum_{l=1}^{k} \mu_{l} p_{l} \\
\text { for all } 1 \leq k \leq K-1 \text { and } \\
\sum_{l=1}^{K} u_{l}(\boldsymbol{p})=\log \left(\frac{\rho \alpha_{K}}{\mu_{K}}\right)-\left[1-\frac{\mu_{K}}{\rho \alpha_{K}}-\sum_{l=1}^{K-1} \frac{\mu_{K} \alpha_{l}}{\alpha_{K}}\right]^{+} \\
-\sum_{l=1}^{K-1} \mu_{l} p_{l} .
\end{array}
$$

Note that utility $u_{K}$ is a function of the utilities $u_{1}, \ldots, u_{K-1}$. Denote the utility region described by (17) or (18) as $\mathcal{U}$, i.e.,

$$
\mathcal{U}=\bigcup_{p \in \mathcal{P}} \boldsymbol{u}(p)
$$

For the two-user case, the parameterization is illustrated in Figure 5 below.

\section{B. Weighted Sum Utility Maximization - SISO MAC}

Consider the following weighted sum utility maximization problem

$$
\max _{\boldsymbol{p} \geq 0} \sum_{k=1}^{K} \lambda_{k} u_{k}(\boldsymbol{p})
$$

with $\lambda_{k} \geq 0$ for $1 \leq k \leq K$ and $\sum_{k=1}^{K} \lambda_{k}=1$. From the discussion around problem (15), we know that the sum utility is maximized if only the best user transmits. Assume the optimal decoding order induced by $\frac{\alpha_{1}}{\mu_{1}} \leq \frac{\alpha_{2}}{\mu_{2}} \leq \ldots \leq \frac{\alpha_{K}}{\mu_{K}}$, it follows that

$$
\lambda_{1} \geq \lambda_{2} \geq \ldots \geq \lambda_{K} .
$$

For all other weights, the maximum sum utility solution solves also (19). In [20] it is shown that users with larger weights $\lambda_{k}$ should be decoded later. There, the weights correspond to the buffer occupancy. The inequalities in (20) induce the same decoding order $K \rightarrow K-1 \rightarrow \ldots \rightarrow 1$ induced by $\frac{\alpha_{1}}{\mu_{1}} \leq \frac{\alpha_{2}}{\mu_{2}} \leq \ldots \leq \frac{\alpha_{K}}{\mu_{K}}$.
1) Characterization of the Optimal Power Allocation: In the following result, we characterize the optimal power allocation which solves the weighted sum utility maximization. Based on this characterization, an efficient algorithm is developed which solves the problem (19) in $K$ steps.

Theorem 2: The optimal power allocation $\boldsymbol{p}^{\prime}$ which solves (19) satisfies for all $0 \leq \ell \leq K-1$

$$
\begin{aligned}
\sum_{l=1}^{K-\ell} \alpha_{l} p_{l}^{\prime} & =\left(\frac{c_{K-\ell}}{\frac{\lambda_{K-\ell} \mu_{K-\ell}}{\alpha_{K-\ell}}-\frac{\lambda_{K-\ell+1} \mu_{K-\ell+1}}{\alpha_{K-\ell+1}}}-\frac{1}{\rho}\right)^{+} \\
& =\phi(\ell),
\end{aligned}
$$

where $c_{k}=\left(\lambda_{k}-\lambda_{k+1}\right)$ with $\lambda_{K+1}=0$ by definition. Furthermore, the following special cases can be distinguished:

1) All users allocate zero power if and only if

$$
\left(\frac{\alpha_{K}}{\mu_{K}}-\frac{1}{\rho}\right) \leq 0
$$

2) For all inverse noise variance $\rho \leq \bar{\rho}$ only user $K$ allocates non-zero power, i.e., we operate in the so called single-user optimality range,

$$
\bar{\rho}=\frac{\left(\frac{\lambda_{K-1} \mu_{K-1}}{\alpha_{K-1}}-\frac{\lambda_{K} \mu_{K}}{\alpha_{K}}\right)}{\lambda_{K-1}-\lambda_{K}} .
$$

3) There does not exists any single-user optimality range at all, i.e., $\bar{\rho}=0$, if and only if

$$
\frac{\lambda_{K-1} \mu_{K-1}}{\alpha_{K-1}}-\frac{\lambda_{K} \mu_{K}}{\alpha_{K}} \leq 0 .
$$

Remark 3: Interestingly, the all zero power condition in (22) does not depend on the weights $\lambda$.

Proof: The first step is to rewrite (19) as a convex programming problem using the Pareto optimal decoding order and the assumption on the order of the weights in (20) as

$$
\sum_{k=1}^{K} \lambda_{k} u_{k}(\boldsymbol{p})=\sum_{k=1}^{K} c_{k} \log \left(1+\rho \sum_{l=1}^{k} \alpha_{l} p_{l}\right)-\sum_{k=1}^{K} \lambda_{k} \mu_{k} p_{k} .
$$

Hence, the non-negative sum of a concave function with respect to $\boldsymbol{p}$ and the sum of linear functions with respect to the power vector $\boldsymbol{p}$ results in a concave function. The constraint set is convex, a feasible point exists and therefore, the KarushKuhn-Tucker (KKT) optimality conditions are necessary and sufficient for all $1 \leq \kappa \leq K$

$$
\begin{array}{r}
\sum_{k=\kappa}^{K} c_{k} \frac{\rho \alpha_{\kappa}}{1+\rho \sum_{l=1}^{k} \alpha_{l} p_{l}}=\lambda_{\kappa} \mu_{\kappa}-\psi_{\kappa}, \quad \psi_{\kappa} p_{\kappa}=0, \\
p_{\kappa} \geq 0, \quad \psi_{\kappa} \geq 0,
\end{array}
$$

where $\psi_{\kappa}$ is the Lagrangian multiplier for the non-negativeness constraint. Evaluate the optimality conditions (26) for $\kappa=K$ to obtain

$$
\frac{c_{K} \rho \alpha_{K}}{1+\rho \sum_{l=1}^{K} \alpha_{l} p_{l}}=\lambda_{K} \mu_{K}
$$

Solving for $\rho \sum_{l=1}^{K} \alpha_{l} p_{l}$ and note that $c_{K}=\lambda_{K}$ yields

$$
\sum_{l=1}^{K} \alpha_{l} p_{l}=\left(\frac{\alpha_{K}}{\mu_{K}}-\frac{1}{\rho}\right)^{+} .
$$


In the following, assume that $\frac{\alpha_{K}}{\mu_{K}} \geq \frac{1}{\rho}$. Otherwise, all users would allocate zero power. Furthermore, evaluate the optimality (26) for $\kappa=K-1$ to obtain

$$
\frac{c_{K-1} \rho \alpha_{K-1}}{1+\rho \sum_{l=1}^{K-1} \alpha_{l} p_{l}}+\frac{c_{K} \rho \alpha_{K-1}}{1+\rho \sum_{l=1}^{K} \alpha_{l} p_{l}}=\lambda_{K-1} \mu_{K-1} \text {. }
$$

Insert (27) into (28) to obtain

$$
\frac{c_{K-1} \rho \alpha_{K-1}}{1+\rho \sum_{l=1}^{K-1} \alpha_{l} p_{l}}=\lambda_{K-1} \mu_{K-1}-c_{K} \frac{\alpha_{K-1}}{\alpha_{K}} \mu_{K} .
$$

Solve for $\rho \sum_{l=1}^{K-1} \alpha_{l} p_{l}$ to obtain

$$
\rho \sum_{l=1}^{K-1} \alpha_{l} p_{l}=\left(\frac{c_{K-1} \rho \alpha_{K-1}}{\lambda_{K-1} \mu_{K-1}-c_{K} \frac{\alpha_{K-1}}{\alpha_{K}} \mu_{K}}-1\right)^{+} .
$$

If the expression in bracket on the right-hand-side (RHS) of (29) is negative, zero power is allocated to users $1, \ldots, K-$ 1 and user $K$ is the only active. From (29), the single-user optimality range in (23) follows by solving for $\rho$.

Now, we consider $\kappa=K-\ell$ for arbitrary $0 \leq \ell \leq K-1$. For this choice of $\kappa$, it follows from (26) that it holds

$$
\begin{array}{r}
\frac{\rho c_{K-\ell}}{1+\rho \sum_{l=1}^{K-\ell} \alpha_{l} p_{l}}+\frac{\rho c_{K-\ell+1}}{1+\rho \sum_{l=1}^{K-\ell+1} \alpha_{l} p_{l}}+\ldots \\
+\frac{\rho c_{K-1}}{1+\rho \sum_{l=1}^{K-1} \alpha_{l} p_{l}}+\frac{\rho c_{K}}{1+\rho \sum_{l=1}^{K} \alpha_{l} p_{l}}=\frac{\lambda_{K-\ell} \mu_{K-\ell}}{\alpha_{K-\ell}} .
\end{array}
$$

For $\kappa=K-\ell+1$, (26) yields

$$
\begin{aligned}
\frac{\rho c_{K-\ell+1}}{1+\rho \sum_{l=1}^{K-\ell+1} \alpha_{l} p_{l}}+\ldots & +\frac{\rho c_{K-1}}{1+\rho \sum_{l=1}^{K-1} \alpha_{l} p_{l}} \\
+\frac{\rho c_{K}}{1+\rho \sum_{l=1}^{K} \alpha_{l} p_{l}} & =\frac{\lambda_{K-\ell+1} \mu_{K-\ell+1}}{\alpha_{K-\ell+1}} .
\end{aligned}
$$

Subtracting (31) from (30) we obtain

$$
\frac{\rho c_{K-\ell}}{1+\rho \sum_{l=1}^{K-\ell} \alpha_{l} p_{l}}=\frac{\lambda_{K-\ell} \mu_{K-\ell}}{\alpha_{K-\ell}}-\frac{\lambda_{K-\ell+1} \mu_{K-\ell+1}}{\alpha_{K-\ell+1}} .
$$

Solving (32) for $\sum_{l=1}^{K-\ell} \alpha_{l} p_{l}$ and taking the Lagrangian multipliers for non-negativeness of $p_{1}, \ldots, p_{K-\ell}$ into account we obtain

$$
\begin{aligned}
\sum_{l=1}^{K-\ell} \alpha_{l} p_{l} & =\left(\frac{c_{K-\ell}}{\frac{\lambda_{K-\ell} \mu_{K-\ell}}{\alpha_{K-\ell}}-\frac{\lambda_{K-\ell+1} \mu_{K-\ell+1}}{\alpha_{K-\ell+1}}}-\frac{1}{\rho}\right)^{+} \\
& =\phi(\ell)
\end{aligned}
$$

The optimal power allocation $p_{1}^{\prime}, \ldots, p_{K}^{\prime}$ has to fulfill the necessary and sufficient optimality conditions and thereby for all $0 \leq \ell \leq K-1$ the equation (33). This completes the proof.

\section{2) Algorithm for Weighted Sum Utility Maximization:}

Based on the characterization in Theorem 2, the following algorithm described in Algorithm 1 solves (19).

The algorithm 1 works as follows. It checks first the allpower off condition from Theorem 2 in (22). Then, power to user $K-\ell$ is computed as the difference between $\frac{\phi(\ell)}{\alpha_{\ell}}$ and $\frac{\phi(\ell+1)}{\alpha_{\ell}}$. Then, it starts with $\ell=0$ and verifies that the $\ell+1$ user optimality condition is not satisfied, e.g., for $\ell=0$ it is (23) in Theorem 2. After at most $K$ steps the algorithm stops and returns the optimal power allocation in $\boldsymbol{p}^{\prime}$.

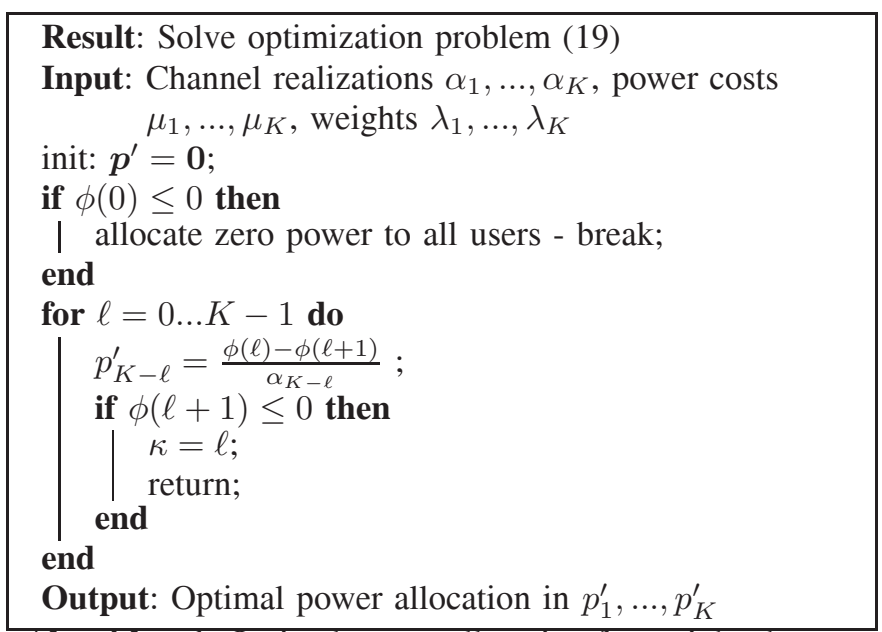

Algorithm 1: Optimal power allocation for weighted sum utility maximization of SISO MAC.

\section{Convexity of the Utility Region of SISO MAC}

The next result shows that the utility region of the SISO MAC is convex.

Theorem 3: The SISO MAC utility region characterized in (17) is convex. For two utility tuples $\boldsymbol{u}=\left[u_{1}, \ldots, u_{K}\right]$ and $\boldsymbol{v}=$ $\left[v_{1}, \ldots, v_{K}\right]$ inside the utility region, the linear combination $\boldsymbol{u}(\tau)=\tau \boldsymbol{u}+(1-\tau) \boldsymbol{v}$ with $0 \leq \tau \leq 1$ is also inside the utility region, i.e.,

$$
\boldsymbol{u} \in \mathcal{U}, \boldsymbol{v} \in \mathcal{U} \Longrightarrow \boldsymbol{u}(\tau) \in \mathcal{U}
$$

Proof: We will prove Theorem 3 in two steps. In the first step, we show that uniqueness of solution of weighted sum utility maximization implies convexity. In the second step, we show that the solution to the weighted sum utility maximization problem in (19) is unique. Denote the convex closure of $\mathcal{U}$ as $\tilde{\mathcal{U}}$. This corresponds to the linear hull of the extreme points with respect to the weighted sum utility.

1) If $\max _{\boldsymbol{u} \in \mathcal{U}} \sum_{k=1}^{K} \lambda_{k} u_{k}$ has a unique solution $\boldsymbol{u}^{*}(\boldsymbol{\lambda})$ for

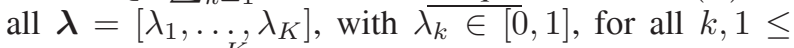
$k \leq K$ with $\sum_{k=1}^{K} \lambda_{k}=1$ then the region $\mathcal{U}$ defined in (17) or (18) is strict convex.

We show this by contradiction: Assume that $\mathcal{U}$ is not strict convex. Then there are at least two $\boldsymbol{u}^{(1)}$ and $\boldsymbol{u}^{(2)}$ (extreme points) such that some part of $\boldsymbol{u}(\tau)=(1-$ $\tau) \boldsymbol{u}^{(1)}+\tau \boldsymbol{u}^{(2)}$ for $0 \leq \tau \leq 1$ is not in $\mathcal{U}$, i.e., $\boldsymbol{u}(\tau) \notin \mathcal{U}$ for some $\tau$. It follows that there are intervals $\left[\tau_{1}, \tau_{2}\right]$ with $\tau_{1}<\tau_{2}$ for which $\boldsymbol{u}(\tau)$ does not belong to the boundary of $\tilde{\mathcal{U}}$. Then, there exists a vector $\lambda>0$ such that

$$
\boldsymbol{u}(\tau) \in \arg \max _{\boldsymbol{u} \in \tilde{\mathcal{U}}} \sum_{k=1}^{K} \lambda_{k} u_{k}
$$

leads to

$$
\begin{aligned}
\max _{\boldsymbol{u} \in \tilde{\mathcal{U}}} \sum_{k=1}^{K} \lambda_{k} u_{k} & =\max _{\boldsymbol{u} \in \mathcal{U}} \sum_{k=1}^{K} \lambda_{k} u_{k}=\sum_{k=1}^{K} \lambda_{k} u_{k}^{(1)} \\
& =\sum_{k=1}^{K} \lambda_{k} u_{k}^{(2)}
\end{aligned}
$$

which contradicts the uniqueness of the solution. 


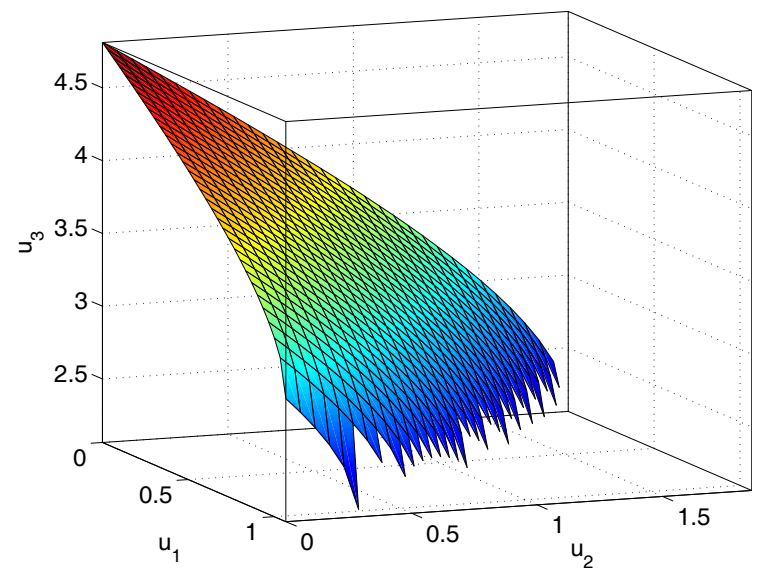

Fig. 6. Three-user MAC utility region for fixed channel realization and $\mu_{1}=\mu_{2}=\mu_{3}=0.01$.

2) The solution to (19) is unique.

The programming problem in (19) is a strictly concave maximization problem over convex constraint set (see the Proof of Theorem 2). Therefore, there must be a unique global optimum. The iterative Algorithm 1 can easily locate the unique global optimum.

For the three-user case, the parameterization is illustrated in Figure 6 below. The convexity of the utility region proved in Theorem 3 can be clearly observed.

\section{SIMO MAC}

If the base station has multiple receive antennas, the optimal SIC order is far more difficult to characterize. Therefore, we begin by investigating the special case when we have two users. Denote the channels between the mobiles and the BS as $\boldsymbol{h}_{1}, \ldots, \boldsymbol{h}_{K}$.

\section{A. Two User Case}

Theorem 4: The optimal power allocation for the two user case and decoding order $1 \rightarrow 2$ is given by

$$
\begin{aligned}
& 0 \leq p_{2} \leq\left(\frac{1}{\mu_{2}}-\frac{1}{\rho\left|\boldsymbol{h}_{2}\right|^{2}}\right)^{+}, \\
& p_{1}=\left(\frac{1}{\mu_{1}}-\frac{1}{\rho\left|\boldsymbol{h}_{1}\right|^{2}-\kappa_{1}}\right)^{+}
\end{aligned}
$$

where $\kappa_{1}=\frac{\rho^{2} p_{2}\left|\boldsymbol{h}_{1}^{H} \boldsymbol{h}_{2}\right|^{2}}{1+\rho p_{2}\left|\boldsymbol{h}_{2}\right|^{2}}$. The optimal power allocation for the decoding order $2 \rightarrow 1$ is given by

$$
\begin{aligned}
& 0 \leq p_{1} \leq\left(\frac{1}{\mu_{1}}-\frac{1}{\rho\left|\boldsymbol{h}_{1}\right|^{2}}\right)^{+}, \\
& p_{2}=\left(\frac{1}{\mu_{2}}-\frac{1}{\rho\left|\boldsymbol{h}_{2}\right|^{2}-\kappa_{2}}\right)^{+}
\end{aligned}
$$

where $\kappa_{2}=\frac{\rho^{2} p_{1}\left|\boldsymbol{h}_{1}^{H} \boldsymbol{h}_{2}\right|^{2}}{1+\rho p_{1}\left|\boldsymbol{h}_{1}\right|^{2}}$.

Proof: The proof for the decoding order $1 \rightarrow 2$ is shown. The proof for the other decoding order is analogue. Denote the utility of user one for decoding order $1 \rightarrow 2$ as $u_{1,1}$. The utility of user one can be written as

$$
u_{1,1}(\boldsymbol{p})=\log \operatorname{det}\left(\boldsymbol{I}+\rho p_{1} \boldsymbol{Z}_{1}^{-1} \boldsymbol{h}_{1} \boldsymbol{h}_{1}^{H}\right)-\mu_{1} p_{1},
$$

with $\boldsymbol{Z}_{1}=\left[\boldsymbol{I}+\rho p_{2} \boldsymbol{h}_{2} \boldsymbol{h}_{2}^{H}\right]$. By the matrix-inversion lemma $\boldsymbol{Z}_{1}^{-1}=\left[\boldsymbol{I}+\rho p_{2} \boldsymbol{h}_{2} \boldsymbol{h}_{2}^{H}\right]^{-1}=\boldsymbol{I}-\frac{\rho p_{2} \boldsymbol{h}_{2} \boldsymbol{h}_{2}^{H}}{1+\rho p_{2}\left|\boldsymbol{h}_{2}\right|^{2}}$ we obtain the utility

$$
u_{1,1}(\boldsymbol{p})=\log \left(1+\rho\left|\boldsymbol{h}_{1}\right|^{2} p_{1}-\frac{\rho p_{1} p_{2}\left|\boldsymbol{h}_{1}^{H} \boldsymbol{h}_{2}\right|^{2}}{1+\rho p_{2}\left|\boldsymbol{h}_{2}\right|^{2}}\right)-\mu_{1} p_{1} .
$$

Next, the upper bound for the power $p_{2}$ is obtained from the maximum of $u_{2,1}\left(p_{2}\right)$ by

$$
\frac{\partial u_{2,1}\left(p_{2}\right)}{\partial p_{2}}=\frac{\rho\left|\boldsymbol{h}_{2}\right|^{2}}{1+\rho\left|\boldsymbol{h}_{2}\right|^{2} p_{2}}-\mu_{2}=0
$$

which leads to

$$
0 \leq p_{2} \leq\left(\frac{1}{\mu_{2}}-\frac{1}{\rho\left|\boldsymbol{h}_{2}\right|^{2}}\right)^{+}
$$

The optimal power $p_{1}$ is now obtained from (36) as a function of $p_{2}$ by setting the derivative with respect to $p_{1}$ to zero, i.e.,

$$
\frac{\partial u_{1,1}(\boldsymbol{p})}{\partial p_{1}}=\frac{\rho\left|\boldsymbol{h}_{1}\right|^{2}-\kappa_{1}}{1+\rho\left|\boldsymbol{h}_{1}\right|^{2} p_{1}-p_{1} \kappa_{1}}-\mu_{1}=0
$$

where $\kappa_{1}=\frac{\rho^{2} p_{2}\left|\boldsymbol{h}_{2}^{H} \boldsymbol{h}_{1}\right|^{2}}{1+\rho p_{2}\left|\boldsymbol{h}_{2}\right|^{2}}$. Solving (38) for $p_{1}$, we obtain the optimal power allocation

$$
p_{1}=\left(\frac{1}{\mu_{1}}-\frac{1}{\rho\left|\boldsymbol{h}_{1}\right|^{2}-\kappa_{1}}\right)^{+}
$$

which completes the proof.

From the result follows the set of achievable utilities denoted by $u_{1,1}, u_{1,2}$ for user one and decoding order $1 \rightarrow 2$ and $2 \rightarrow 1$, respectively, and $u_{2,1}, u_{2,2}$ for user two and for the two decoding orders.

Corollary 2: The utilities on the Pareto boundary for fixed decoding order $1 \rightarrow 2$ are

$$
\begin{aligned}
u_{1,1}\left(p_{2}\right)= & \log \left(\frac{\rho\left|\boldsymbol{h}_{1}\right|^{2}-\kappa_{1}}{\mu_{1}}\right)^{+}- \\
& {\left[1-\frac{\mu_{1}}{\rho\left|\boldsymbol{h}_{1}\right|^{2}-\kappa_{1}}\right]^{+} } \\
u_{2,1}\left(p_{2}\right)= & \log \left(1+\rho\left|\boldsymbol{h}_{2}\right|^{2} p_{2}\right)-\mu_{2} p_{2} .
\end{aligned}
$$

For fixed decoding order $2 \rightarrow 1$ they are

$$
\begin{aligned}
u_{1,2}\left(p_{1}\right)= & \log \left(1+\rho\left|\boldsymbol{h}_{1}\right|^{2} p_{1}\right)-\mu_{1} p_{1} \\
u_{2,2}\left(p_{1}\right)= & \log \left(\frac{\rho\left|\boldsymbol{h}_{2}\right|^{2}-\kappa_{2}}{\mu_{2}}\right)^{+}- \\
& {\left[1-\frac{\mu_{2}}{\rho\left|\boldsymbol{h}_{2}\right|^{2}-\kappa_{2}}\right]^{+} . }
\end{aligned}
$$

Remark 4: In the optimal power allocation as well as in the utility expressions the impact of the spatial correlation of the channels $\boldsymbol{h}_{1}$ and $\boldsymbol{h}_{2}$ can be clearly observed

$$
0 \leq \kappa_{1,2} \leq \frac{\rho^{2} p_{2}\left|\boldsymbol{h}_{1}\right|\left|\boldsymbol{h}_{2}\right|}{1+\rho p_{2}\left|\boldsymbol{h}_{2}\right|^{2}}
$$

where the lower bound is achieved for completely uncorrelated channels $\boldsymbol{h}_{1}$ and $\boldsymbol{h}_{2}$.

The characterization of the Pareto boundary is illustrated in Figure 7 below. 


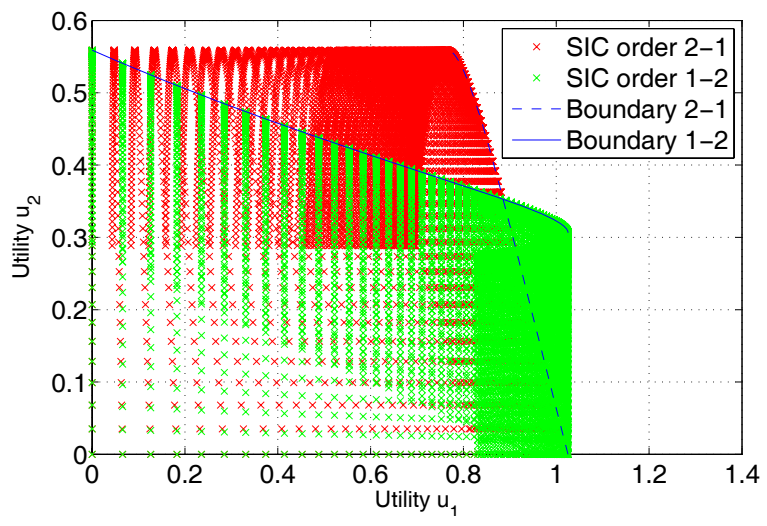

Fig. 7. Two-user SIMO MAC utility region and its Pareto boundary for fixed channel realization and SNR $0 \mathrm{~dB}$.

\section{B. Sum Utility Maximization - SIMO MAC}

The sum utility of the SIMO MAC with SIC (arbitrary decoding order) reads

$$
u_{s}(\boldsymbol{p})=\log \operatorname{det}\left(\boldsymbol{I}+\rho \sum_{k=1}^{K} p_{k} \boldsymbol{h}_{k} \boldsymbol{h}_{k}^{H}\right)-\sum_{k=1}^{K} \mu_{k} p_{k} .
$$

The corresponding programming problem

$$
\max _{\boldsymbol{p} \geq 0} u_{s}(\boldsymbol{p})
$$

is a convex optimization problem and standard interior point methods could be used to find the optimal power allocation vector. In the next section, we will propose an iterative waterfilling type of algorithm to solve the sum utility maximization of the MIMO MAC. This algorithm could be obviously used to solve (43) using power $p_{k}$ instead of transmit covariance matrices $Q_{k}$ for inner optimization. However, in the single transmit antenna case, we can characterize the optimal power allocation for low SNR. First, we convert the programming problem in (44) into a familiar representation. Define

$$
q_{k}=p_{k} \mu_{k} \quad \longrightarrow \quad p_{k}=\frac{q_{k}}{\mu_{k}}
$$

and define $\sum_{k=1}^{K} q_{k}=Q$. Then the programming problem (44) is equivalent to

$$
\max _{Q \geq 0} \max _{\substack{q_{k} \geq 0 \\ \sum_{k=1}^{K} q_{k} \leq Q}} \log \operatorname{det}\left(\boldsymbol{I}+\rho \sum_{l=1}^{K} q_{k} \tilde{\boldsymbol{h}}_{k} \tilde{\boldsymbol{h}}_{k}^{H}\right)-Q
$$

with effective channel $\tilde{\boldsymbol{h}}_{k}=\frac{\boldsymbol{h}_{k}}{\sqrt{\mu_{k}}}$. Note that the inner maximization is similar to the SIMO MAC sum rate maximation with sum power constraint [21]. Next, we are interested in a characterization of the optimal power allocation by the parameter range in which only one user is active, i.e., $p_{1}>0$ and $p_{2}=\ldots=p_{K}=0$.

Theorem 5: If the users are ordered according to

$$
\frac{\left|\boldsymbol{h}_{1}\right|^{2}}{\mu_{1}} \geq \frac{\left|\boldsymbol{h}_{2}\right|^{2}}{\mu_{2}} \geq \ldots \geq \frac{\left|\boldsymbol{h}_{K}\right|^{2}}{\mu_{K}}
$$

then there is a range - the so called single-user range - of $0 \leq \rho \leq \bar{\rho}$ such that $\mu_{1} \leq \bar{\rho}\left|\boldsymbol{h}_{1}\right|^{2}$ and

$$
\bar{\rho}=\mu_{1}\left(\frac{\left|\boldsymbol{h}_{1}\right|^{2} \mu_{2}-\left|\boldsymbol{h}_{2}\right|^{2} \mu_{1}}{\left|\boldsymbol{h}_{1}\right|^{2}\left|\boldsymbol{h}_{2}\right|^{2}-\left|\boldsymbol{h}_{1}^{H} \boldsymbol{h}_{2}\right|^{2}}+\frac{1}{\left|\boldsymbol{h}_{1}\right|^{2}}\right)
$$

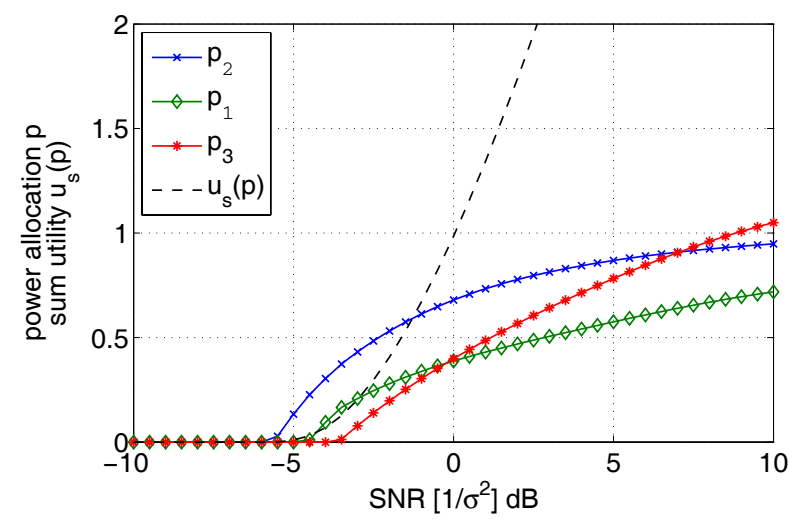

Fig. 8. Three-user SIMO MAC sum utility and corresponding optimal power allocation. Single-user optimality range $\bar{\rho}=-4.5632 \mathrm{~dB}$ as given in (48).

such that only one user is active with the optimal power allocation

$$
p_{1}=\left(\frac{1}{\mu_{1}}-\frac{1}{\rho\left|\boldsymbol{h}_{1}\right|^{2}}\right) .
$$

Proof: As in [21, Theorem 6], we sort the users according to their effective channels

$$
\left|\tilde{\boldsymbol{h}}_{1}\right|^{2} \geq\left|\tilde{\boldsymbol{h}}_{2}\right|^{2} \geq \ldots \geq\left|\tilde{\boldsymbol{h}}_{K}\right|^{2}
$$

which is the same order as in (47). Then the result says that for fixed $Q \geq 0$, only the first user is supported, i.e., $q_{1}=Q$ and $q_{2}=\ldots=q_{K}=0$, if and only if

$$
\tilde{\boldsymbol{h}}_{1}^{H}\left[\boldsymbol{I}+\rho Q \tilde{\boldsymbol{h}}_{1} \tilde{\boldsymbol{h}}_{1}^{H}\right]^{-1} \tilde{\boldsymbol{h}}_{1} \geq \tilde{\boldsymbol{h}}_{2}^{H}\left[\boldsymbol{I}+\rho Q \tilde{\boldsymbol{h}}_{1} \tilde{\boldsymbol{h}}_{1}^{H}\right]^{-1} \tilde{\boldsymbol{h}}_{2} .
$$

Using the matrix-inversion lemma again, it follows from (50)

$$
\left|\tilde{\boldsymbol{h}}_{1}\right|^{2}-\left|\tilde{\boldsymbol{h}}_{2}\right|^{2} \geq \rho Q\left(\left|\tilde{\boldsymbol{h}}_{1}\right|^{2}\left|\tilde{\boldsymbol{h}}_{2}\right|^{2}-\left|\tilde{\boldsymbol{h}}_{1}^{H} \tilde{\boldsymbol{h}}_{2}\right|^{2}\right) .
$$

On the other hand, in the single-user range, we have the optimal power allocation

$$
Q^{*}=\left(\frac{1}{\mu_{1}}-\frac{1}{\rho\left|\boldsymbol{h}_{1}\right|^{2}}\right) .
$$

Using the optimal single-user power allocation $Q^{*}$ from (52) in (51) leads to the desired single-user optimality range in (48).

Let us illustrate the single-user optimality range in Figure 8. Three users with power prices $\boldsymbol{\mu}=[1.2,1,0.8]$, four receive antennas and three channel realizations given by

$$
\left.\begin{array}{ccc} 
& \multicolumn{2}{c}{\boldsymbol{H}=\left[\boldsymbol{h}_{1}, \boldsymbol{h}_{2}, \boldsymbol{h}_{3}\right]=} \\
-0.73-0.08 i & 0.51+0.01 i & -0.31-0.06 i \\
-0.54+0.09 i & 0.02+0.57 i & 0.02-0.72 i \\
1.53-0.56 i & 1.08+0.49 i & -0.22+0.66 i \\
0.30-0.16 i & -1.20-0.16 i & 0.69-0.80 i
\end{array}\right)
$$

are used to compute the optimal power allocation (using the iterative single-user optimization outline in the MIMO MAC section) and the corresponding maximum sum utility. The optimal user order for this set of channels and power prices is $2 \rightarrow 1 \rightarrow 3$ for small SNR.

One interesting observation is that the order of the users regarding their power allocation changes completely from 
small $\operatorname{SNR} p_{2} \geq p_{1} \geq p_{3}$ to high $\operatorname{SNR} p_{3} \geq p_{1} \geq p_{2}$. For high SNR, the optimal power allocation converges to $\frac{1}{\mu_{k}}$, in the example above this corresponds to

$$
p_{1} \rightarrow \frac{1}{1.2} \approx 0.833, \quad p_{2} \rightarrow 1, \quad p_{3} \rightarrow 1.25 .
$$

The low- and high- SNR characterization corresponds well with the behavior of the power allocation observed in Figure 8. In the next section, we allow the transmitter to have multiple antennas and study the sum utility maximization of the MIMO MAC.

\section{MIMO MAC}

In the MIMO MAC, each multiple antenna transmitter can optimize its transmit covariance matrix $\boldsymbol{Q}_{k}$ including its overall transmit power $\operatorname{tr}\left(\boldsymbol{Q}_{k}\right)$.

In [22], an iterative waterfilling algorithm is proposed to solve the sum capacity maximization of the MIMO MAC. The idea is to perform alternating optimization by keeping all users transmit covariance matrices fixed expect user $l$ and optimize the utility with respect to user $l$. This is done iteratively until the algorithm converges. The convergence follows because the objective is increased in each step and it is concave and bounded. Therefore, the iterative algorithm must converge. A generalization to a broader class of utility functions is developed in [21]. In [23], an algorithm based on dual decomposition is developed to solve the sum rate maximization for the Gaussian broadcast channel. The power prices $\mu_{k}$ correspond exactly to the dual variables.

However, the iterative waterfilling algorithm and the dual decomposition approach cannot be applied to the weighted sum utility maximization problem. Therefore, we present here an alternative approach. Consider the following programming problem

$$
\begin{aligned}
\max _{\boldsymbol{Q}_{k} \succeq 0} & \sum_{k=1}^{K} \lambda_{k} \log \operatorname{det}\left(\boldsymbol{I}+\rho \sum_{l=1}^{k} \boldsymbol{H}_{l} \boldsymbol{Q}_{l} \boldsymbol{H}_{l}^{H}\right) \\
& -\lambda_{k} \log \operatorname{det}\left(\boldsymbol{I}+\rho \sum_{l=1}^{k-1} \boldsymbol{H}_{l} \boldsymbol{Q}_{l} \boldsymbol{H}_{l}^{H}\right) \\
& -\lambda_{k} \mu_{k} \operatorname{tr}\left(\boldsymbol{Q}_{k}\right) .
\end{aligned}
$$

Define $\lambda_{K+1}=0$, the objective function can be reformulated as

$$
\begin{array}{r}
\sum_{k=1}^{K}\left(\lambda_{k}-\lambda_{k+1}\right) \log \operatorname{det}\left(\boldsymbol{I}+\rho \sum_{l=1}^{k} \boldsymbol{H}_{l} \boldsymbol{Q}_{l} \boldsymbol{H}_{l}^{H}\right) \\
-\sum_{k=1}^{K} \lambda_{k} \mu_{k} \operatorname{tr}\left(\boldsymbol{Q}_{k}\right) .
\end{array}
$$

The objective function in (55) is jointly concave in $Q_{1}, \ldots, Q_{K}$ and therefore the algorithm developed in [24] can be adapted. The main difference is that here we do not have a trace constraint on $\boldsymbol{Q}_{k}$ and thus do not need the normalization step.

The convergence of Algorithm 2 can be shown following similar arguments as in [24]. If $\mu_{k}$ in Algorithm 2 is chosen as $\operatorname{tr}\left(\boldsymbol{Q}_{k}\right)$ then it corresponds to the normalization step in [24]. The convergence rate of the fixed point optimization algorithm is only linear [25] and any Newton style algorithm has local super-linear convergence. However, it provides a simple universal approach to weighted sum rate maximization.
Result: Solve weighted sum utility optimization problem

Input: Channel realizations $\boldsymbol{H}_{1}, \ldots, \boldsymbol{H}_{K}$, power costs $\mu_{1}, \ldots, \mu_{K}$, inverse noise power $\rho$.

init: $\boldsymbol{Q}_{k}=\mathbf{0}$ for all $1 \leq k \leq K$;

while required accuracy not reached do

$$
\begin{aligned}
\boldsymbol{Q}_{k}= & \frac{1}{\mu_{k} \lambda_{k}} \sum_{l=k}^{K}\left(\lambda_{l}-\lambda_{l+1}\right) \rho \boldsymbol{Q}_{k}^{1 / 2} \boldsymbol{H}_{k}^{H} \\
& \cdot\left[\boldsymbol{I}+\sum_{m=1}^{l} \boldsymbol{H}_{m} \boldsymbol{Q}_{m} \boldsymbol{H}_{m}^{H}\right]^{-1} \boldsymbol{H}_{k} \boldsymbol{Q}_{k}^{1 / 2}
\end{aligned}
$$

Output: Optimal $\boldsymbol{Q}_{1}, \ldots, \boldsymbol{Q}_{K}$

Algorithm 2: Weighed sum utility maximization for MIMO MAC.

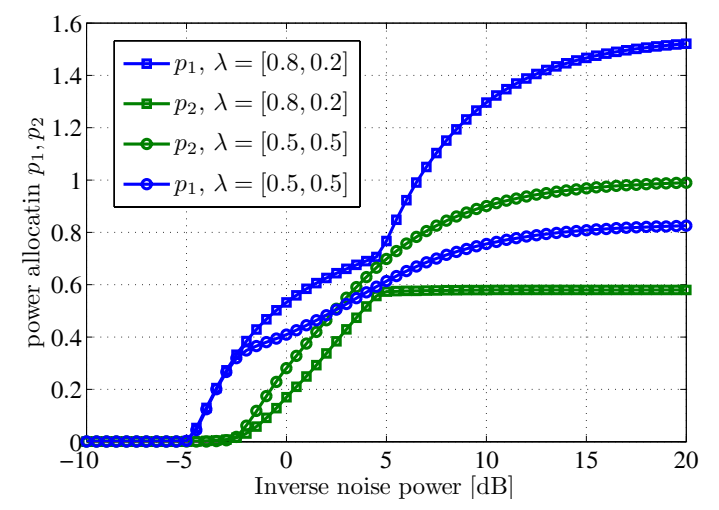

Fig. 9. Two-user MIMO $\left(n_{T}=2, n_{R}=2\right)$ MAC optimal weighted sum utility power allocation.

Corollary 3: The Algorithm 2 converges always to a global optimal set of transmit covariance matrices $Q_{1}, \ldots, Q_{K}$.

\section{A. Illustration of MIMO MAC Weighted Sum Utility Maxi- mization}

In Figure 9, the optimal power allocation is shown for a twouser MIMO $\left(n_{t}=2, n_{R}=2\right)$ MAC with fixed but random channel matrices and power prices $\boldsymbol{\mu}=[1.2,1]$. The weights are $\lambda_{1}=0.8, \lambda_{2}=0.2$. The sum utility optimization $\lambda_{1}=$ $\lambda_{2}=0.5$ is shown for comparison.

Furthermore, the difference to the case with single antenna transmitters can be clearly observed. At different points $\rho$ multiple eigenmodes of the two transmitters are activated. Therefore, the order of the allocated power varies. Note that for sum utility maximization asymptotically, the allocated power of user $l$ converges to $\frac{n_{T}}{\mu_{l}}$. In general, the quantitative behavior of the optimal power allocation is difficult to characterize for medium SNR values because it depends on the spatial signatures of the involved users and their weights.

\section{CONCLUSION}

We proposed a utility function for energy-aware resource allocation and transmit optimization in multiuser uplink systems based on the difference of the individual capacity and 
a weighted power penalty term. For the SISO MAC, we completely characterized the utility region and proposed an efficient algorithm to achieve any point on the Pareto boundary. The region is always convex. However, in the SIMO MAC, the utility region is no longer convex due to the additional spatial degrees of freedom and due to the different decoding orders at the base station. In the MIMO MAC case, we extended the iterative waterfilling algorithm to solve the maximum sum utility problem. The important question, how to choose the prices in order to optimize system utility functions is left for future research. The decentralized implementation of the two proposed algorithms is another interesting research direction.

\section{ACKNOWLEDGMENT}

The authors would like to thank Marcin Wiczanowski and Zhijiat Chong for helpful discussions.

\section{REFERENCES}

[1] "Sustainable energy use in mobile communications," ERICSSON White Paper, Tech. Rep., 2007.

[2] V. Rodoplu and T. H. Meng, "Minimum energy mobile wireless networks," IEEE J. Sel. Areas Commun., vol. 17, pp. 1333-1344, 1999.

[3] L. Li and J. Y. Halpern, "Minimum-energy mobile wireless networks revisited," in Proc. IEEE ICC, vol. 1, 2001, pp. 278-283.

[4] V. Rodoplu and T. H. Meng, "Bits-per-joule capacity of energy-limited wireless networks," IEEE Trans. Wireless Commun., vol. 6, pp. 857-865, 2007.

[5] X. Huang and I. Rubin, "Bit-per-joule performance of power saving ad hoc networks with a mobile backbone," Wireless Netw., 2009.

[6] L. Li, N. B. Shroff, and R. Srikant, "Energy-aware routing in sensor networks: a large system approach," Ad Hoc Netw., vol. 5, pp. 818-831, 2007.

[7] C.-H. Min and S. Kim, "On-demand utility-based power control routing for energy-aware optimization in mobile ad hoc networks," Wireless Personal Commun., vol. 41, pp. 259-280, Apr. 2007.

[8] Z. Zhou, S. Zhou, J.-H. Cui, and S. Cui, "Energy-efficient cooperative communication based on power control and selective single-relay in wireless sensor networks," IEEE Trans. Wireless Commun., vol. 7, pp. 3066-3078, 2008

[9] B. Bougard, G. Lenoir, A. Dejonghe, L. V. Der Perre, F. Catthoor, and W. Dehaene, "SMARTMIMO: an energy-aware adaptive MIMO-OFDM radio link control for next-generation wireless local area networks," EURASIP J. Wireless Commun. Netw., pp. article ID 98 186, 2007.

[10] P. Grover and A. Sahai, "Green codes: energy-efficient short-range communication," in Proc. IEEE ISIT, pp. 1178-1182, 2008.

[11] D. Acemoglu, A. Ozdaglar, and R. Srikant, "The marginal user principle for resource allocation in wireless networks," in Proc. IEEE Conf. Dec. Control, vol. 2, pp. 1544-1549, 2004.

[12] F. Meshkati, M. Chiang, H. V. Poor, and S. C. Schwartz, "A gametheoretic approach to energy-efficient power control in multicarrier CDMA systems," IEEE J. Sel. Areas Commun., vol. 24, pp. 1115-1129, 2006.

[13] D. Tse and S. Hanly, "Multiaccess fading channels—part I: polymatroid structure, optimal resource allocation and throughput capacities," IEEE Trans. Inf. Theory, vol. 44, pp. 2796-2815, Nov. 1998.

[14] B. Rimoldi and R. Urbanke, "A rate-splitting approach to the Gaussian multiple-access channel," IEEE Trans. Inf. Theory, vol. 42, pp. 364-375, 1996.

[15] C. U. Saraydar, N. B. Mandayam, and D. J. Goodman, "Efficient power control via pricing in wireless data networks," IEEE Trans. Commun., vol. 50, pp. 291-303, 2002.

[16] W. Yu and J. M. Cioffi, "Sum capacity of Gaussian vector broadcast channels," IEEE Trans. Inf. Theory, vol. 50, pp. 1875-1892, 2004.

[17] S. Gunturi and F. Paganini, "Game theoretic approach to power control cellular CDMA," in Proc. IEEE VTC, 2003.

[18] J. Duplicy, J. Louveaux, and L. Vandendorpe, "Utility-based MIMO uplink beamforming," in Proc. IEEE Workshop SAMSP, pp. 254-257, 2006.

[19] S. Boyd and L. Vandenberghe, Convex Optimization. Cambridge University Press, 2004.

[20] H. Boche and M. Wiczanowski, "Optimization-theoretic analysis of stability-optimal transmission policy for multiple antenna multiple access channel," IEEE Trans. Signal Process., vol. 55, pp. 2688-2702, 2007.
[21] H. Boche and E. A. Jorswieck, "On the performance optimization in multiuser MIMO systems," European Trans. Telecommun., vol. 18, pp. 217-233, Apr. 2006.

[22] W. Yu, W. Rhee, S. Boyd, and J. M. Cioffi, "Iterative water-filling for Gaussian vector multiple-access channels," IEEE Trans. Inf. Theory, vol. 50, pp. 145-151, 2004.

[23] W. Yu, "Sum capacity computation for the Gaussian vector broadcast channel via dual decomposition," IEEE Trans. Inf. Theory, vol. 52, pp. 754-759, Feb. 2006.

[24] E. Jorswieck, B. Ottersten, A. Sezgin, and A. Paulraj, "Feedback reduction in uplink MIMO OFDM systems by chunk optimization," in Proc. IEEE ICC, 2008.

[25] C. T. Kelly, Iterative Methods for Linear and Nonlinear Equations. SIAM, 1995.

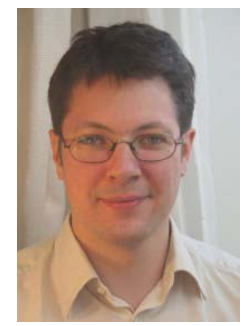

Eduard A. Jorswieck (S'01-M'05-SM'08) received his Diplom-Ingenieur degree and DoktorIngenieur (Ph.D.) degree, both in electrical engineering and computer science from the Berlin University of Technology (TUB), Germany, in 2000 and 2004, respectively. He was with the Fraunhofer Institute for Telecommunications, Heinrich-HertzInstitute (HHI) Berlin, from 2001 to 2006. In 2006, he joined the Signal Processing Department at the Royal Institute of Technology (KTH) as a post-doc and became a Assistant Professor in 2007. Since February 2008, he has been the head of the Chair of Communications Theory and Full Professor at Dresden University of Technology (TUD), Germany.

His research interests are within the areas of applied information theory, signal processing and wireless communications. He is senior member of IEEE and elected member of the IEEE SPCOM Technical Committee. From 2008-2010 he serves as an Associate Editor for IEEE SIGNAL PROCESSINC LETTERS. In 2006, he was co-recipient of the IEEE Signal Processing Society Best Paper Award.

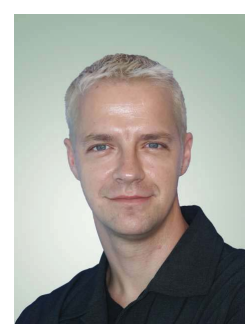

Holger Boche (M'04 - SM'07) received his Dipl.Ing. and Dr.-Ing. degrees in Electrical Engineering from the Technische Universitaet Dresden, Germany, in 1990 and 1994, respectively. In 1992 he graduated in Mathematics from the Technische Universitaet Dresden, and in 1998 he received his Dr.rer.nat. degree in pure mathematics from the Technische Universitaet Berlin. From 1994 to 1997, he did postgraduate studies in mathematics at the Friedrich-Schiller Universität Jena, Germany. In 1997, he joined the Heinrich-Hertz-Institut (HHI) für Nachrichtentechnik Berlin. Since 2002, he has been Full Professor for Mobile Communication Networks at the Technische Universität Berlin at the Institute for Communications Systems. In 2003, he became Director of the Fraunhofer German-Sino Lab for Mobile Communications, Berlin, Germany, and since 2004 he has also been Director of the Fraunhofer Institute for Telecommunications (HHI), Berlin, Germany. He was Visiting Professor at the ETH Zurich during winter term 2004 and 2006 and at KTH Stockholm during summer term 2005. Prof. Boche received the Research Award "Technische Kommunikation" from the Alcatel SEL Foundation in October 2003, the "Innovation Award" from the Vodafone Foundation in June 2006, Gottfried Wilhelm Leibniz Prize from the Deutsche Forschungsgemeinschaft (German Research Foundation) in 2008. He was co-recipient of the 2006 IEEE Signal Processing Society Best Paper Award and recipient of the 2007 IEEE Signal Processing Society Best Paper Award. He is a member of IEEE Signal Processing Society SPCOM and SPTM Technical Committee. He was elected a member of the German Academy of Sciences (Leopoldina) in 2008 and of Berlin Brandenburg Academy of Sciences and Humanities in 2009.

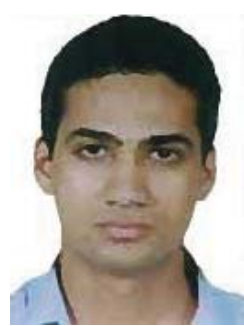

Siddharth Naik received his MS in EE from the Royal Institute of Technology in 2004. He was a research engineer at the SPANN laboratory at IIT, Mumbai in 2004. He has been a research associate at the Heinrich Hertz Institute and has been pursuing his $\mathrm{PhD}$ in Electrical Engineering at the Technical University of Berlin, since 2006. His research interests are game theory, mechanism design, probability theory and convex optimization. 